\title{
Stabilising Touch Interactions in Cockpits, Aerospace, and Vibrating Environments
}

\author{
B. I. Ahmad ${ }^{\dagger}$, P. M. Langdon ${ }^{\dagger \dagger}$ and S. J. Godsill ${ }^{\dagger}$ \\ ${ }_{\dagger}$ Signal Processing and Communications Laboratory (SigProC) \\ $\dagger^{\dagger}$ Engineering Design Centre (EDC) \\ Department of Engineering, University of Cambridge, UK \\ \{bia23, pml24, sjg30\}@cam.ac.uk
}

\begin{abstract}
Incorporating touch screen interaction into cockpit flight systems is increasingly gaining traction given its several potential advantages to design as well as usability to pilots. However, perturbations to the user input are prevalent in such environments due to vibrations, turbulence and high accelerations. This poses particular challenges for interacting with displays in the cockpit, for example, accidental activation during turbulence or high levels of distraction from the primary task of airplane control to accomplish selection tasks. On the other hand, predictive displays have emerged as a solution to minimize the effort as well as cognitive, visual and physical workload associated with using in-vehicle displays under perturbations, induced by road and driving conditions. This technology employs gesture tracking in 3D and potentially eye-gaze as well as other sensory data to substantially facilitate the acquisition (pointing and selection) of an interface component by predicting the item the user intents to select on the display, early in the movements towards the screen. A key aspect is utilising principled Bayesian modelling to incorporate and treat the present perturbation, thus, it is a software-based solution that showed promising results when applied to automotive applications. This paper explores the potential of applying this technology to applications in aerospace and vibrating environments in general and presents design recommendations for such an approach to enhance interactions accuracy as well as safety.
\end{abstract}

Keywords: Interactive displays, Bayesian inference, target assistance, turbulence.

\section{Introduction}

In the development of aviation cockpit interfaces, a key step has been the movement from conventional mechanical controls (such as joysticks, trackballs, dials, switches, levers, and buttons [1]), and analogue displays to electronic "glass cockpits". This facilitated the introduction of touch screens into cockpits. They bring several advantages to the manufactures as well as operators (e.g. pilots) due to their ability to:

1) offer additional design flexibilities by combining the display-input-feedback functionalities in one module, simplifying complex dial arrays and providing better visibility, salience and flexibility of display of critical flight information,

2) promote intuitive interactions via free hand pointing gestures, 
3) incorporate large quantities of information associated with modern aviation developments (e.g. weather radar, collision avoidance, route control, ALS, autopilot and numerous information related to engine parameters and other on-board systems); amongst others this led to the reductions of space required for the cockpit bulkhead as well as control area, and

4) being easily updatable via reconfiguring the Graphical User Interfaces (GUIs) in lieu of incurring the prohibitive overheads of repurposing-rewiring hardware panels of mechanical controls.

Thereby, touch screens use in aerospace applications is gaining traction [2-8] and can be viewed as the predominant design choice for modern cockpit HMI, e.g. F-35 Joint Strike Fighter has a full-panel-width $20 \times 8$ inches touch screen.

However, air turbulence, accelerations (e.g. constant high G-force) and possibly vibrations due to taxiway roughness can significantly affect the ability of an operator to accomplish on-display target acquisition (pointing and selection) tasks via pointing gestures $[3,4,8,9]$. This is contrary to classical physical controls of non-touchscreen displays (e.g. joystick, rotary, etc.), which offer mechanical stabilisation of the pointing action, especially that touch screen devices as input technology cannot present discreet button regions and can only be activated by contact with the screen. Accordingly, perturbations could result in erroneous user input and/or unintentional (false) selections. Attempts to rectify such incorrect selections, e.g. by repeating the acquisition task(s), can tie up further of the operator's attention, which can be otherwise available for more essential tasks such as controlling the jet and monitoring its on-board systems. Therefore, interacting with displays under perturbations (e.g. turbulence and vibrations) via pointing gestures can be highly effortful-distracting and a key challenge is to develop solutions to notably improve the usability and performance (e.g. accuracy) of touch interactions in aerospace and general vibrating environments [3-9].

On the other hand, touch screens are an integrated part of the modern vehicle environment with several established benefits, similar to those of listed above [10,11]. However, undertaking a free hand pointing gesture to acquire a target on the display, e.g. a GUI icon, requires devoting a considerable amount of attention (visual, cognitive and physical) that would be instead dedicated to the primary task of driving [12], with potential safety implications [13]. Due to road and driving conditions, the user pointing gesture can be subject to perturbations (e.g. vibrations and lateral accelerations) leading to substantial degradation in the touch accuracy and interaction performance $[12,14]$. Therefore, the predictive touch technology $[15,16]$, which can infer, notably early in the free hand pointing gesture, the intended on-screen item has emerged as an effective solution that can simplify and expedite the selection task, even under perturbations. It can significantly improve the usability of in-car touch screens by reducing distractions and workload associated with interacting with them, under various road and driving conditions.

In this paper, we first consider existing approaches to stabilising the user input in cockpits and then propose applying the predictive touch technology, originally developed for automotive applications, as well as statistical filtering techniques as in [16, 17]. The aim is to substantially improve interactions accuracy as well as performance and usability of touch screens where perturbations (e.g. due to turbulence, vibrations 
and high accelerations) can be prevalent and carrying out the "secondary" tasks of interacting with various in-cockpit controls can be highly demanding. Design considerations and recommendations to best utilise the predictive display or touch technology are also presented.

The remainder of this paper is organised as follows. In the next section, we consider existing work in Human-Computer Interaction (HCI); in particular that related to counteracting the impact of perturbations on the user input (finger pointing) in cockpits. We then give a brief overview of the proposed predictive display technology and the adopted Bayesian framework, outlining the flexibilities of this formulation and its requirements in Section 3. In Section 4, a few design recommendations are highlighted and finally conclusions are drawn in Section 5.

\section{Related Work and Desired Features}

\subsection{Related Work}

With the proliferation of the increasingly ubiquitous touch screen technology in everyday use, target acquisition (pointing and selection) on a graphical user interface has become part of modern life and a frequent HCI task. The majority of modern software products now employ touch pads, touch screens and stylus entry systems to permit the user to input information in the wide varieties of formats necessitated by complex internet functionalities. For example, on screen keypads and keyboards, 2D mapping information, 2D drawing and art tools, 3D representations, as well as conventional screen buttons with standard selection paradigms such as select on mouse-up if within the button boundaries. Furthermore, a whole generation of new interaction techniques have been developed based on touch screen contact. This includes simple gestures with one finger and more recent multi-touch gesture displays that allow multiple finger contact for pinch or sweep gestures $[5,18]$. Touch-based interfaces allow a finger or pointing device to be used as a mouse when in contact with the screen. These can be capacitive or resistive (or both) to allow for the use of gloves or use in wet environments.

In the general HCI area, pointing (e.g. with a mouse cursor) reliability and accuracy is of a key importance for the design of effective GUI. This has triggered an immense interest in approaches that model pointing movements and assist the pointing task by reducing the cursor pointing time and improving its accuracy [19-26]. This can be achieved via pointing facilitation techniques, such as increasing the size of the target icon, altering its activation area, dragging the cursor closer to the target, etc. However, such strategies can be effectively applied only if the intended GUI item is known $a$ priori [21-26]. Such studies focus on pointing via a mouse or mechanical-device in a 2D set-up to select a GUI icon(s) and often consider able-bodied computer users in a stationary input situation and do not treat the impact of perturbations on the interactions.

However, the user population is diverse and includes motion impaired, elderly and non-expert users. Similar to users experiencing perturbations induced by environmental factors (e.g. due to road and driving conditions or using a touch screen whilst walking), the pointing-selection task can be challenging for users with a motion-visual impairment [17, 26-30]. One area offering mitigation to these challenges is the design of 
integrated multimodal display and control technologies for ease of input and task completion [17, 27, 30]. Initially, in the domain of better design for elderly and impaired computer and TV users and in the form of extraordinary user interfaces [31, 32]. This approach assumes that any human user can be impaired (disabled) in their effectiveness by characteristics of their environment, the task, and the design of the user interface they are presented with [32].

On the other hand, the problem of improving touch interactions in cockpits under perturbations is not well explored and innovations in this area are rather limited, unlike touch interactions with a portable devices (e.g. smartphones) under situational-induced impairments, for instance whilst walking [33, 34], or under health-induced impairment [26-32]. Existing approaches to combat the effects of present perturbations in cockpits can be divided into the following two general categories:

1- Selection Strategy: utilise a suitable criteria to establish a trigger for the selection action of a GUI item from finger(s) touch, e.g. location of on-screen mouse up/down event, dwell time on a particular interface item, double tap, pressure applied during a touch action and others [35]. This is a well-known issue in the area of HCI and plethora of establish approaches; a selection strategy is an integrated part of most touch screen UI design (including those for displays with multi-touch capabilities). Nonetheless, under perturbations such strategies cannot often effectively differentiate between intentional and noise-induced accidental contacts with the display [36, 37].

2- Braced touch interaction: allows users to mechanically stabilise selections by bracing their fingers/hand/palm on or around the touch screen. For instance, a bezel edge can be used as in [8] or placing one or more of the hand fingers on the display and utilising eye-gaze fixations on the touched GUI item for ensuring an intentional touch as in [38]. They aim to enable accurate target selection (e.g. using an adequate selection strategy) during high levels of vibration, without impeding interaction performance when vibrations are absent. Such approach face the following key challenges include: a) discriminating intentional selection from braced contacts and b) limited interaction area which is often confined to the vicinity of the brace, especially for wide interactive displays, rendering various regions of the touch screen inaccessible. Mitigating the latter often requires moving the hand into awkward postures and/or uncomfortable selection maneuvers since one or more of the hand fingers are used for stabilisation.

\subsection{Desired Features}

In addition to improving the performance of the cockpit display in terms of accuracy (e.g. minimising erroneous selections) and given the constraints of a typical aerospace environment, a suitable solution should possess the following features:

> Context dependent: it is expected to be applicable to a wide range of possible scenarios and cockpit control functionalities. It thus should effectively captures the different accuracy requirements under various conditions, e.g. safety critical control commands versus an interaction to obtain an update on a particular status.

$>$ Adaptable: the characteristics of the target acquisition task can be affected by many factors, including the operator's physical ability, prior experience and level of experienced perturbations. The adopted solution should be also independent or easily 
adaptable to various GUI layout design and able to make use of any available priors on the user's behaviour to refine its performance.

$>$ Reduces the interactions effort: the adopted solution should reduce the workload (manual, visual, cognitive) and inattention associated with accomplishing a selection task, unlike with the braced touch interaction. Thereby, it minimises distractions from other critical tasks such as jet control, on-board systems monitoring, etc.

As illustrated below, the proposed predictive technology meets these requirements.

\section{Predictive Touch: Intent Inference with Hidden Markov Models}

The predictive touch system block diagram is depicted in Figure 1. Its main aim is to predict the icon the user intends to select, i.e. $\mathcal{D}_{I}$, as early as possible in the free hand pointing gesture; it then facilitates-expedites the target selection albeit the present perturbations. This system utilises a gesture tracker and other sensory data in conjunction with probabilistic inference algorithms to determine the intended destination on the interactive surface (e.g. touch screen). The prediction results for each of the GUI selectable icons are subsequently used to decide on the intended endpoint and accordingly alter the GUI to assist the selection process. Next, we describe the main building blocks of a predictive touch system.

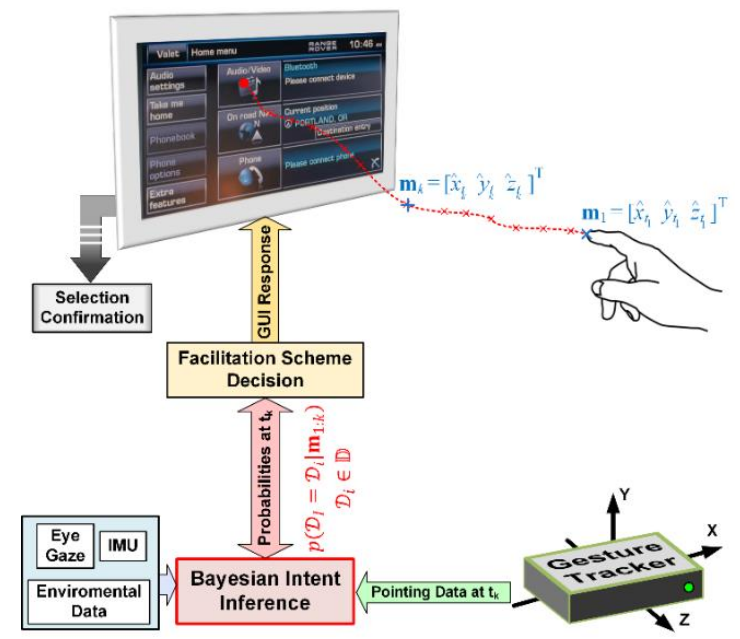

Figure 1. System block diagram with a complete pointing finger-tip trajectory; $\mathbf{m}_{k}$ is the finger-tip Cartesian 3D coordinates at time $t_{k}$ where $t_{k}>t_{1}[16]$.

\subsection{Pointing Gesture Tracker}

It provides, in real-time, the pointing hand/finger(s) locations in 3D, for example at the discrete time instants $t_{1}, t_{2}, \ldots, t_{k}$. Several such pointing gesture trackers, which can accurately track, in real-time, a pointing gesture in 3D, have emerged lately. They are motivated by a desire to extend HCI beyond traditional keyboard input and mouse pointing. Whilst some trackers are vision-based [39], such as Microsoft Kinect, Leap 
Motion controller and Nimble UX, others are more amenable to being incorporated into wearables, e.g. [40-43], and thus can be more suitable for a cockpit setting.

\subsection{Bayesian Predictor and Statistical Filtering of Noise}

Let $\mathbb{D}=\left\{\mathcal{D}_{i}: i=1,2, \ldots N\right\}$ be the set of $N$ selectable items on the display, for example, GUI selectable icons. This key system module calculates the likelihood of each of the selectable interface items, i.e. $\mathcal{D}_{i} \in \mathbb{D}$, being the intended destination $\mathcal{D}_{I}$ at a given time instant, e.g. $t_{k}$. These probabilities can be expressed by:

$$
\mathcal{P}\left(t_{k}\right)=\left\{p\left(\mathcal{D}_{i}=\mathcal{D}_{I} \mid \mathbf{m}_{1: k}\right), \quad i=1,2, \ldots, N\right\},
$$

such that $\mathbf{m}_{1: k} \triangleq\left\{\mathbf{m}_{1}, \mathbf{m}_{2}, \ldots, \mathbf{m}_{k}\right\}$ are the filtered gesture-tracker observations (can include other sensory data) at the successive time instants $\left\{t_{1}, t_{2}, \ldots, t_{k}\right\}$. For example, $\mathbf{m}_{n}=\left[\begin{array}{lll}\hat{x}_{t_{n}} & \hat{y}_{t_{n}} & \hat{z}_{t_{n}}\end{array}\right]^{\prime}$ is the Cartesian coordinates of the pointing finger at $t_{n}$. It is noted that data sorting, analysis and association steps are needed prior to utilising the pointing gesture data.

The predictor can use a number of low complexity probabilistic models that are amenable to real-time implementations. A review of these models is given in this $[15,16]$, showing that they can lead to a Kalman-filter-type implementation of the intent inference routine. Within the Bayesian framework,

$$
p\left(\mathcal{D}_{I}=\mathcal{D}_{i} \mid \mathbf{m}_{1: k}\right) \propto p\left(\mathbf{m}_{1: k} \mid \mathcal{D}_{I}=\mathcal{D}_{i}\right) p\left(\mathcal{D}_{I}=\mathcal{D}_{i}\right)
$$

where the prior $p\left(\mathcal{D}_{i}=\mathcal{D}_{I}\right)$ on the $i^{\text {th }}$ selectable item (independent of the current pointing task) can be attained from semantic data, frequency of use, other sensory data, etc. This makes the adopted formulation particularly appealing as any additional information (if/when becomes available) can be easily incorporated into the inference process via the priors.

\section{Modelling of Intent with HMMs}

The Bayesian destination predictor applied here relies on defining a Hidden Markov Model (HMM) of the pointing motion in 3D, effectively capturing the intrinsic influence of the intended on-display endpoint (i.e. interface selectable icon) on the finger/hand movements during the pointing gesture $[16,17]$. This is fundamentally distinct from previous HCI research on endpoint prediction in 2D scenarios, which often follow from Fitt's law type analysis and uses deterministic models. The statistical modelling approach employed by a predictive touch system captures the variability among users and their motor capabilities via Stochastic Differential Equations (SDEs), which represent the destination-motivated pointing motion in $3 \mathrm{D}$ or even $2 \mathrm{D}$.

Most importantly, a suitable Bayesian formulation with HMMs also allows the flexible and effective modelling of the pointing motion with perturbations via the SDEs. The variability in the pointing movement, e.g. due to the user behavior and/or perturbations, can be introduced through the noise element of the state (position, velocity, acceleration, etc.) evolution equation. Additionally, the noise generated from the employed sensor, e.g. a particular gesture tracker, can be incorporated via the measurement 
noise in the observation equation. For further details on the modelling aspect of predictive touch, including destination-driven ones and bridged distributions, the reader is referred to $[15,16,44,45]$.

\section{Removing Perturbations: Smoothing Noisy Trajectories}

Employing Gaussian and linear motion as well as observation models permits using the efficient Kalman filters to determine (1); typically $N$ or more such filters are needed $[15,16]$. When the user input is perturbed, the predictive display system can handle noisy $3 \mathrm{D}$ pointing gestures by setting the noise covariance in the motion model relative to the measured (experienced) perturbations. This conforms with the Gaussian and linear modelling assumptions and a higher covariance corresponds to having less certainty in the estimated destination-driven pointing finger position, velocity, etc. This technique is suitable for low to medium perturbation levels that can be represented by Gaussian noise, e.g. driving on smooth to moderately bumpy-paved roads.

The assumption of Gaussian noise in a motion model can be overly restrictive in a highly perturbed environments since the pointing hand/finger can move in a highly erratic manner. It can exhibit sudden unintentional noise-related jump movements or jolts. In such scenarios, the perturbations present can be treated as an additional nonlinear random jump process causing sudden large changes in the pointing finger position and velocity. For example, this can be modelled by the mean-reverting jump-diffusion velocity process $[16,44]$. Likelihood estimation for such motion models relies on sequential Monte Carlo (SMC), particle, filtering [44, 46], which is computationally costly compared to Kalman filtering. A practical alternative to applying this computationally-expensive inference procedure $N$ or more times, is to apply the SMC filtering once as a pre-processing stage prior to the destination prediction routine. The objective of this pre-processing stage is to remove the most severe effects of large jolts from the gesture-tracker observations $\mathbf{m}_{1: k}$ at $t_{k}$ and allow the utilisation of the original linear motion models for intent inference [16]. This approach represents a compromise between effective removal of severe perturbations (jumps) and the computational efficiency of the original models. Figure 2 depicts an example of filtering a highly perturbed pointing trajectory using SMC.

Applying a pre-processing SMC filter or dynamically adjusting the motion model covariance can be guided by additional sensory data, such as changes in the level of experienced perturbations from auxiliary sensors. Additionally, the filtered free-hand pointing gesture can be used not only for pointing, but also for general gesture-based interactions. 


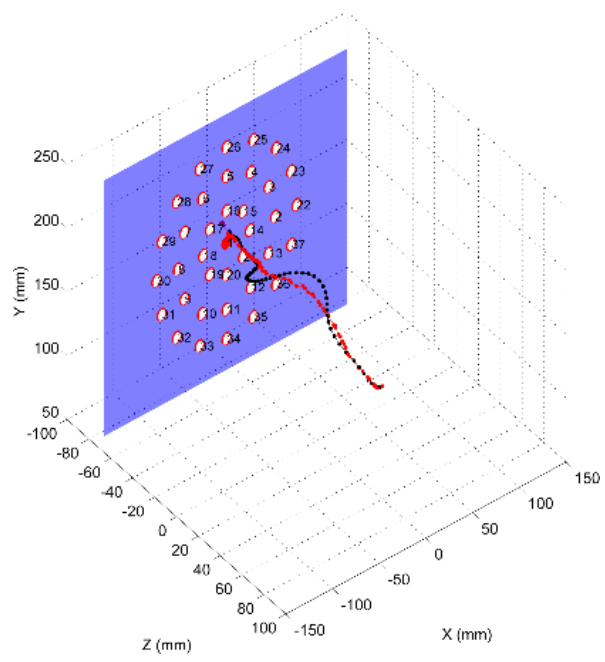

Figure 2. 3D pointing track before (black) and after (red) applying a variable rate particle filter [44].

\subsection{Feedback-Decision Scheme}

Given the inference results $\mathcal{P}\left(t_{k}\right)$ at time $t_{k}$, the GUI is modified (if applicable) to facilitate accomplishing the selection task. For example, the system expands/colours the GUI items based on $\mathcal{P}\left(t_{k}\right)$ or select the predicted intended on-screen item on behalf of the user, i.e. without the user physically touching the display surface. The latter is dubbed mid-air selection. Possible criterions for deciding the endpoint of the pointing gesture at $t_{k}$ (if needed depending on the feedback scheme) include choosing the most probable destination (i.e. Maximum a Posteriori estimate), the selectable GUI icon whose probability exceeds a certain threshold and many others. The decision making process within the Bayesian formulation can be addressed, namely in relation to minimising: $\mathbb{E}_{\mathcal{D}_{I}}\left[\mathcal{C}\left(\mathcal{D}^{*}, \mathcal{D}_{I}\right) \mid \mathbf{m}_{1: k}\right]$ where $\mathcal{C}\left(\mathcal{D}^{*}, \mathcal{D}_{I}\right)$ is the cost of deciding $\mathcal{D}^{*}$ at $t_{k}$ as the on-screen endpoint given that $\mathcal{D}_{I}$ is the true intended destination.

\subsection{Additional Sensory Data}

The availability of additional sensory data such as inertia measurement unit (accelerometer/gyroscope) can enable the system to determine the operating conditions (e.g. whether the user input is noisy) and accordingly modify the applied model and/or adapt its parameters and/or perform smoothing prior to intent prediction. On the other hand, eye-gaze can provide valuable information on the areas of interest on the display. If such information become available, they can be easily incorporated into the Bayesian framework via the priors $\operatorname{Pr}\left(\mathcal{D}_{i}=\mathcal{D}_{I}\right), i=1,2, \ldots, N$. Alternatively, additional data can be part of the observations/measurements vectors $\mathbf{m}_{1: k}$. 


\subsection{Final Remarks}

In summary, the developed Bayesian framework for predictive touch enables:

- Handling varying levels of perturbations in the pointing gesture, including those induced by the variability in the pointing behaviour, environment-induced noise (e.g. turbulence, vibrations and accelerations).

- Catering for varying levels of noise due to the sensory technology and inaccuracies in the collected measurements (e.g. inaccurate pointing finger and eye-gaze locations).

- Treatment of irregularly spaced and asynchronous data (e.g. due to the sensory technology) since continuous-time dynamics and observation models are utilised.

- Incorporating contextual information (GUI design, frequency of use, user profile, context as well as history of use, etc.).

- Principled fusion of various sensory data (when available) that can assist facilitating interactions with the touch screen.

Thereby, it meets the desired featured outlined in Section 2.

\section{Design Recommendations}

The main design recommendations stem from the capabilities of the predictive touch approach, enabled by intent prediction. For instance, its robustness with respect to perturbation and applying intrinsic filtering and principled a Bayesian statistical approach. In fact, it is capable of delivering accurate predictions within 30-50 percent of a movement towards the screen as shown from on-road experimental studies for automotive applications $[16,47]$. It is effective when the following criteria are present:

1. for perturbed environments, including in the presence of extreme jumpsjolts in the pointing movement,

2. where tracking data is incomplete, unevenly timed, erratic, and often incorrect (i.e. noisy),

3. for displays or any region of space within the tracking volume that the user may move towards, including non-screen elements (dials, switches, etc.),

4. where reduction of workload or physical stretching is required.

From a design perspective, any device that can offer 3D tracking of user pointing movement and a display may use this approach. For example, a mobile device such as a tablet whilst walking. Unlike bracing approaches or force dependent interactions which are ergonomically inconvenient in a cockpit environment, this technology facilitates usability of interactive displays in general including those that do not have a physical surface to touch (e.g. non-touch displays, 3D projections and HUDs) via mid-air selection and where screens are inaccessible. 


\section{Conclusions}

Consideration of touch screen displays in aviation environments shows that significant issues exist in preventing interaction errors as a result of accidental selection, failure to select, or incorrect selection resulting from external perturbations on both the user and the screen. Three possible solutions to this problem were discussed, namely: 1) utilizing suitable selection strategies (e.g. pressure-based selection), 2) braced interaction, and 3) predictive touch with Bayesian intent prediction. Unlike the former two, the predictive interactive display within a Bayesian framework is well suited to the human centred design of new information-rich and multimodal interfaces in aerospace applications. It can effectively incorporate variabilities in the environment as well as interaction styles, contextual information and additional sensory data (when available), within the stochastic pointing movement and measurement models as well as the modelling priors. Thus, the developed predictive displays framework is a promising approach to achieving substantial significant usability improvements to interactions in cockpits and vibrating environments. Nevertheless, future experimental work is required to substantiate its benefits in aerospace applications.

Intentionality prediction from the tracked pointing movement has the key advantages that Bayesian prediction can be made without and before contact with the screen, reducing workload, and filtering out (suppressing) unintentional perturbationsinduced pointing movements (e.g. resulting from vibrations and accelerations). However, a disadvantage of this technology is that the operator's pointing movements (e.g. in 3-D) must be tracked. Nonetheless, plethora of third party solutions (gesture tracking technologies) are now available for use in confined spaces including automotive and aerospace, see [39-43]. This gives rise to a technology that is potentially not restricted to screens of any sort and may also assist or allow free-air gestures in a perturbed vibrating environment.

\section{Acknowledgements}

This work was carried out under the University of Cambridge CAPE agreement with Jaguar Land Rover.

\section{References}

[1] Stanton, N. A., Harvey, C., Plant, K. L., and Bolton, L. (2013). To twist, roll, stroke or poke? A study of input devices for menu navigation in the cockpit. Ergonomics, 56(4), 590-611.

[2] ARINC661. Cockpit Display System Interfaces to User Systems. ARINC Specification 661, Supplement 6. Airlines Electronic Engineering Committee (AEEC), 216.

[3] Avsar, H., Fischer, J. E., and Rodden, T. (2016, September). Designing touch-enabled electronic flight bags in SAR helicopter operations. In Proceedings of the International Conference on Human-Computer Interaction in Aerospace (p. 14). ACM. 
[4] Dodd, S., Lancaster, J., Miranda, A., Grothe, S., DeMers, B., and Rogers, B. (2014, September). Touch screens on the flight deck: The impact of touch target size, spacing, touch technology and turbulence on pilot performance. In Proceedings of the Human Factors and Ergonomics Society Annual Meeting (Vol. 58, No. 1, pp. 6-10). Sage CA: Los Angeles, CA: SAGE Publications.

[5] Hamon, A., Palanque, P., Andre, R., Barboni, E., Cronel, M., and Navarre, D. (2014, July). Multi-Touch interactions for control and display in interactive cockpits: issues and a proposal. In Proceedings of the International Conference on Human-Computer Interaction in Aerospace (p. 7). ACM.

[6] Kaminani, S. (2011, October). Human computer interaction issues with touch screen interfaces in the flight deck. In Digital Avionics Systems Conference (DASC), 2011 IEEE/AIAA 30th (pp. 6B4-1). IEEE.

[7] Mangion, D. Z., Bécouarn, L., Fabbri, M., and Bader, J. (2011, September). A Single Interactive Display Concept for Commercial and Business Jet Cockpits. In ATIO'2011, Conference of the AIAA on Aviation Technology, Integration, and Operations.

[8] Cockburn, A., Gutwin, C., Palanque, P., Deleris, Y., Trask, C., Coveney, A. and MacLean, K. (2017, May). Turbulent Touch: Touchscreen Input for Cockpit Flight Displays. In Proceedings of the 2017 CHI Conference on Human Factors in Computing Systems (pp. 6742-6753). ACM.

[9] Lin, C. J., Liu, C. N., Chao, C. J., and Chen, H. J. (2010). The performance of computer input devices in a vibration environment. Ergonomics, 53(4), 478-490.

[10] Burnett, G. E., and Porter, J. M. (2001). Ubiquitous computing within cars: designing controls for non-visual use. International Journal of Human-Computer Studies, 55(4), 521-531.

[11] Burnett, G., Lawson, G., Millen, L., and Pickering, C. (2011). Designing touchpad userinterfaces for vehicles: which tasks are most suitable?. Behaviour \& Information Technology, 30(3), 403-414.

[12] Jæger, M. G., Skov, M. B., and Thomassen, N. G. (2008, April). You can touch, but you can't look: interacting with in-vehicle systems. In Proceedings of the SIGCHI Conference on Human Factors in Computing Systems (pp. 1139-1148). ACM.

[13] Klauer, S. G., Dingus, T. A., Neale, V. L., Sudweeks, J. D., and Ramsey, D. J. (2006). The impact of driver inattention on near-crash/crash risk: An analysis using the 100-car naturalistic driving study data. National Highway Traffic Safety Administration, DOT HS 8105942006.

[14] Ahmad, B. I., Langdon, P. M., Godsill, S. J., Hardy, R., Skrypchuk, L., and Donkor, R. (2015, September). Touchscreen usability and input performance in vehicles under different road conditions: An evaluative study. In Proceedings of the 7th International Conference on Automotive User Interfaces and Interactive Vehicular Applications (pp. 4754). ACM.

[15] Ahmad, B. I., Murphy, J. K., Langdon, P. M., Godsill, S. J., Hardy, R., and Skrypchuk, L. (2016). Intent inference for hand pointing gesture-based interactions in vehicles. IEEE Transactions on Cybernetics, 46(4), 878-889.

[16] Ahmad, B. I., Murphy, J. K., Godsill, S., Langdon, P. M., and Hardy, R. (2017). Intelligent interactive displays in vehicles with intent prediction: A Bayesian framework. IEEE Signal Processing Magazine, 34(2), 82-94. 
[17] Langdon, P., Godsill, S., and Clarkson, P. J. (2006). Statistical estimation of user's intentions from motion impaired cursor use data. Proceedings of the 6th International Conference on Disability, Virtual Reality and Associated Technologies (ICDVRAT 2006), Esbjerg, Denmark.

[18] Benko, H., Wilson, A. D., and Baudisch, P. (2006, April). Precise selection techniques for multi-touch screens. In Proceedings of the SIGCHI conference on Human Factors in computing systems (pp. 1263-1272). ACM.

[19] MacKenzie, I. S. (1992). Fitts' law as a research and design tool in human-computer interaction. Human-computer interaction, 7(1), 91-139.

[20] Kopper, R., Bowman, D. A., Silva, M. G., \& McMahan, R. P. (2010). A human motor behavior model for distal pointing tasks. International journal of human-computer studies, 68(10), 603-615.

[21] McGuffin, M. J., \& Balakrishnan, R. (2005). Fitts' law and expanding targets: Experimental studies and designs for user interfaces. ACM Transactions on Computer-Human Interaction (TOCHI), 12(4), 388-422.

[22] Murata, A. (1998). Improvement of pointing time by predicting targets in pointing with a PC mouse. International Journal of Human-Computer Interaction, 10(1), 23-32.

[23] Wobbrock, J. O., Fogarty, J., Liu, S. Y. S., Kimuro, S., \& Harada, S. (2009, April). The angle mouse: target-agnostic dynamic gain adjustment based on angular deviation. In Proceedings of the SIGCHI Conference on Human Factors in Computing Systems (pp. 1401-1410). ACM.

[24] Asano, T., Sharlin, E., Kitamura, Y., Takashima, K., \& Kishino, F. (2005, October). Predictive interaction using the delphian desktop. In Proceedings of the 18th annual ACM symposium on User interface software and technology (pp. 133-141). ACM.

[25] Lank, E., Cheng, Y. C. N., \& Ruiz, J. (2007, April). Endpoint prediction using motion kinematics. In Proceedings of the SIGCHI conference on Human Factors in Computing Systems(pp. 637-646). ACM.

[26] Ahmad, B. I. Langdon, P. Bunch P. and S. Godsill. Probabilistic Intentionality Prediction for Target Selection Based on Partial Cursor Tracks, In Proceedings of the 8th International Conference on Universal Access in Human-Computer Interaction (UAHCI 2014), Heraklion, Crete, Greece (June 22-27, 2014). Lecture Notes in Computer Science, vol. 8515. Berlin, Germany: Springer, pp. 427-438.

[27] Keates, S., Hwang, F., Langdon, P., Clarkson, P. J., \& Robinson, P. (2002, July). Cursor measures for motion-impaired computer users. In Proceedings of the fifth international ACM conference on Assistive technologies (pp. 135-142). ACM.

[28] Gajos, K. Z., Wobbrock, J. O., \& Weld, D. S. (2007, October). Automatically generating user interfaces adapted to users' motor and vision capabilities. In Proceedings of the 20th annual ACM symposium on User interface software and technology (pp. 231-240).

[29] Domingo, M. C. (2012). An overview of the Internet of Things for people with disabilities. Journal of Network and Computer Applications, 35(2), 584-596.

[30] Biswas, P., \& Langdon, P. (2012). Developing multimodal adaptation algorithm for mobility impaired users by evaluating their hand strength. International Journal of HumanComputer Interaction, 28(9), 576-596.

[31] Newell, A. F., \& Gregor, P. (1997). Human computer interfaces for people with disabilities. In Handbook of Human-Computer Interaction (Second Edition) (pp. 813-824). 
[32] Sears, A., Lin, M., Jacko, J., \& Xiao, Y. (2003, June). When computers fade: Pervasive computing and situationally-induced impairments and disabilities. In HCI International (Vol. 2, No. 03, pp. 1298-1302).

[33] Ng, A., Williamson, J., and Brewster, S. (2015, August). The effects of encumbrance and mobility on touch-based gesture interactions for mobile phones. In Proceedings of the 17th International Conference on Human-Computer Interaction with Mobile Devices and Services (pp. 536-546). ACM.

[34] Goel, M., Findlater, L., and Wobbrock, J. (2012, May). WalkType: using accelerometer data to accomodate situational impairments in mobile touch screen text entry. In Proceedings of the SIGCHI Conference on Human Factors in Computing Systems (pp. 26872696). ACM.

[35] Zhai, S., Kristensson, P. O., Appert, C., Anderson, T. H., and Cao, X. (2012). Foundational issues in touch-surface stroke gesture design - an integrative review. Foundations and Trends in Human-Computer Interaction, 5(2), 97-205.

[36] Wang, F., Cao, X., Ren, X., and Irani, P. (2009, October). Detecting and leveraging finger orientation for interaction with direct-touch surfaces. In Proceedings of the 22nd annual ACM symposium on User interface software and technology (pp. 23-32). ACM.

[37] Mayer, S., Gad, P., Wolf, K., Woźniak, P. W., and Henze, N. (2017, September). Understanding the ergonomic constraints in designing for touch surfaces. In Proceedings of the 19th International Conference on Human-Computer Interaction with Mobile Devices and Services (p. 33). ACM.

[38] Kawalkar, A. N., and Roth, H. (2017). Touch screen display user interface and method for improving touch interface utility on the same employing a rules-based masking system. U.S. Patent No. 9,733,707. Washington, DC: U.S. Patent and Trademark Office.

[39] Rautaray, S. S., \& Agrawal, A. (2015). Vision based hand gesture recognition for human computer interaction: a survey. Artificial Intelligence Review, 43(1), 1-54.

[40] Lu, Z., Chen, X., Li, Q., Zhang, X., \& Zhou, P. (2014). A hand gesture recognition framework and wearable gesture-based interaction prototype for mobile devices. IEEE transactions on human-machine systems, 44(2), 293-299.

[41] Gupta, H. P., Chudgar, H. S., Mukherjee, S., Dutta, T., \& Sharma, K. (2016). A continuous hand gestures recognition technique for human-machine interaction using accelerometer and gyroscope sensors. IEEE Sensors Journal, 16(16), 6425-6432.

[42] Labrecque, M. (2014, October). Wearable gesture control: Improving the computer interface convenience and accessibility. In Games Media Entertainment (GEM), 2014 IEEE (pp. 1-1). IEEE.

[43] Kim, D., Hilliges, O., Izadi, S., Butler, A. D., Chen, J., Oikonomidis, I., \& Olivier, P. (2012, October). Digits: freehand 3D interactions anywhere using a wrist-worn gloveless sensor. In Proceedings of the 25th annual ACM symposium on User interface software and technology (pp. 167-176). ACM.

[44] Ahmad, B. I., Murphy, J., Langdon, P. M., and Godsill, S. J. (2014, September). Filtering perturbed in-vehicle pointing gesture trajectories: Improving the reliability of intent inference. In IEEE International Workshop on Machine Learning for Signal Processing (MLSP), 2014 (pp. 1-6). IEEE. 
[45] Ahmad, B. I., Murphy, J. K., Langdon, P. M., and Godsill, S. J. (2018). Bayesian intent prediction in object tracking using bridging distributions. IEEE Transactions on Cybernetics, 41(1), 215-227.

[46] Cappé, O., Godsill, S. J., and Moulines, E. (2007). An overview of existing methods and recent advances in sequential Monte Carlo. Proceedings of the IEEE, 95(5), 899-924.

[47] Ahmad, B. I., Langdon, P. M., Godsill, S. J., Donkor, R., Wilde, R., \& Skrypchuk, L. (2016, October). You do not have to touch to select: A study on predictive in-car touchscreen with mid-air selection. In Proceedings of the 8th International Conference on Automotive User Interfaces and Interactive Vehicular Applications (pp. 113-120). ACM. 